Research Article

\title{
A Mixed Discontinuous Galerkin Method for the Helmholtz Equation
}

\author{
Qingjie Hu, ${ }^{1}$ Yinnian He ${ }^{1 D},{ }^{1}$ Tingting $\mathrm{Li}^{2}{ }^{2}$ and Jing Wen ${ }^{1}$ \\ ${ }^{1}$ School of Mathematics and Statistics, Xi'an Jiaotong University, Xi'an, Shaanxi 710049, China \\ ${ }^{2}$ School of Mathematics and Statistics, Henan University, Kaifeng 475004, China \\ Correspondence should be addressed to Yinnian He; heyn@mail.xjtu.edu.cn
}

Received 1 April 2020; Accepted 15 April 2020; Published 4 May 2020

Academic Editor: Yumin Cheng

Copyright (c) 2020 Qingjie Hu et al. This is an open access article distributed under the Creative Commons Attribution License, which permits unrestricted use, distribution, and reproduction in any medium, provided the original work is properly cited.

In this paper, we introduce and analyze a mixed discontinuous Galerkin method for the Helmholtz equation. The mixed discontinuous Galerkin method is designed by using a discontinuous $\mathbf{P}_{p+1}^{-1}-P_{p}^{-1}$ finite element pair for the flux variable and the scattered field with $p \geq 0$. We can get optimal order convergence for the flux variable in both $H$ (div)-like norm and $L^{2}$ norm and the scattered field in $L^{2}$ norm numerically. Moreover, we conduct the numerical experiments on the Helmholtz equation with perturbation and the rectangular waveguide, which also demonstrate the good performance of the mixed discontinuous Galerkin method.

\section{Introduction}

We consider the following nonhomogeneous Helmholtz equation with the Robin boundary condition:

$$
\begin{aligned}
-\Delta u-k^{2} u & =f, \quad \text { in } \Omega \in \mathbf{R}^{d}, \\
\frac{\partial u}{\partial n_{\Omega}}-i k u & =-g, \quad \text { on } \Gamma=\partial \Omega,
\end{aligned}
$$

where $d=2,3, i=\sqrt{-1}$ denotes the imaginary unit, $k \in \mathbf{R}_{+}$is a given positive number and known as the wave number, $\Omega$ is an open and bounded domain, and $f \in L^{2}(\Omega)$ represents a harmonic source. The Robin boundary condition (2) is the lowest-order absorbing boundary condition [1]. The applications of the Helmholtz equation are extensive in many practical applications, such as geophysics and radar detecting, simulation of ground penetrating, biomedical imaging, acoustic noise control, and seismic wave propagation. The numerical solution of the Helmholtz equation is fundamental to the simulation of time harmonic wave phenomena in acoustics, electromagnetics, and elasticity. However, it remains a challenge to design robust and efficient numerical algorithms for the Helmholtz equation, especially with the large wave number or highly oscillatory solution.

In recent years, many numerical methods have been developed to solve and analyze the Helmholtz equation, for instance, finite difference method [2-5], conforming finite element method [6,7], boundary element method [8], weak Galerkin finite element method [9-12], spectral method [13], and adaptive finite element method [14] . It is also well known that the discontinuous Galerkin methods are flexible and highly parallelizable, and hence discontinuous Galerkin methods are widely used to solve the Helmholtz equation numerically, such as interior penalty discontinuous Galerkin method [15], hybridizable discontinuous Galerkin method $[16,17]$, local discontinuous Galerkin method [18], and the references therein. However, the local discontinuous Galerkin method [19-21] is known to be more physical and flexible on designing discontinuous Galerkin schemes. Two local discontinuous Galerkin methods are studied in [18], where the $\mathbf{P}_{1}^{-1}-P_{1}^{-1}$ finite element pair was used to approximate the flux variable and the scattered field. They obtain the suboptimal convergence for the flux variable. In the numerical fluxes, the authors choose the penalty parameter $\beta>0$ in theoretical analysis and numerical experiments. 
In this paper, we set the penalty parameter $\beta=0$ in numerical fluxes and use the discontinuous $\mathbf{P}_{p+1}^{-1}-P_{p}^{-1}(p \geq 0)$ finite element pair to approximate the flux variable and the scattered field. In addition, we obtain the optimal convergence for the scattered field and the flux variable in the $L^{2}$ norm and the flux variable in the $H$ (div)-like norm numerically.

The paper is organized as follows. In Section 2, we give the definition of the numerical fluxes and introduce the mixed discontinuous Galerkin method. A series of numerical experiments are presented to validate the effectiveness of the mixed discontinuous Galerkin method in Section 3. The paper concludes with conclusions in Section 4.

\section{Mixed Local Discontinuous Galerkin Method}

2.1. Meshes and Notations. In order to establish the mixed discontinuous Galerkin method, we first introduce some notations. The standard space, norm, and inner product notations are adopted in this paper. In particular, for a bounded domain $D,(\cdot, \cdot)_{D}$ and $\langle\cdot, \cdot\rangle_{\partial D}$ denote the $L^{2}$ - inner product on complex-valued spaces $L^{2}(D)$ and $L^{2}(\partial D)$, respectively.

Let $\mathrm{T}_{h}$ be a shape regular triangulation of the domain $\Omega$ with mesh size $h, h_{K}$ be the diameter of any $K \in \mathrm{T}_{h}$ and $h=\max _{K \in \mathrm{T}_{h}} h_{K}, \mathscr{E}_{h}$ be the set of all edges in $\mathrm{T}_{h}, \mathscr{E}_{h}^{I}$ be the collection of all interior edges, and $\mathscr{E}_{h}^{B}=\mathscr{E}_{h} / \mathscr{E}_{h}^{I}$ be the set of boundary edges. For an interior edge $e$ shared by two elements $K_{1}$ and $K_{2}, \mathbf{n}_{1}$ and $\mathbf{n}_{2}$ are the unit outward normal vectors of them, respectively. For any scalar function $v$ and vector-valued function $\tau$, let $v_{i}=\left.v\right|_{\partial K_{i}}$ and $\tau_{i}=\left.\tau\right|_{\partial K_{i}}$ $(i=1,2)$, and the average $\{\cdot\}$ and jump $[\cdot]$ are defined as follows:

$$
\begin{aligned}
& \{v\}=\frac{1}{2}\left(v_{1}+v_{2}\right),\{\boldsymbol{\tau}\}=\frac{1}{2}\left(\boldsymbol{\tau}_{1}+\boldsymbol{\tau}_{2}\right), \quad e \in \mathscr{E}_{h}^{I}, \\
& {[v]=v_{1} \mathbf{n}_{1}+v_{2} \mathbf{n}_{2},[\boldsymbol{\tau}]=\boldsymbol{\tau}_{1} \cdot \mathbf{n}_{1}+\boldsymbol{\tau}_{2} \cdot \mathbf{n}_{2}, \quad e \in \mathscr{E}_{h}^{I} .}
\end{aligned}
$$

On a boundary edge $e \in \mathscr{E}_{h}^{B}$, we set

$$
\begin{aligned}
& \{v\}=v, \\
& \{\boldsymbol{\tau}\}=\boldsymbol{\tau}, \\
& {[v]=v \mathbf{n},} \\
& {[\tau]=\boldsymbol{\tau} \cdot \mathbf{n},}
\end{aligned}
$$

where $\mathbf{n}$ is the outward unit normal vector on $\partial \Omega$.

2.2. Mixed Discontinuous Galerkin Scheme. In this section, we introduce the mixed discontinuous Galerkin method for the nonhomogeneous Helmholtz equations (1) and (2). Firstly, we introduce an auxiliary variable $\sigma=-\Delta u$ and rewrite equation (1) into

$$
\begin{aligned}
\boldsymbol{\sigma}+\Delta u=0, & \text { in } \Omega, \\
\operatorname{div} \boldsymbol{\sigma}-k^{2} u=f, & \text { in } \Omega .
\end{aligned}
$$

Next, we introduce the mixed discontinuous Galerkin method to find the solution pair $(u, \sigma)$ of (5) and (6) numerically. Multiplying (5) and (6) by test functions $\bar{\tau}$ and $\bar{v}$, respectively, and integrating both equations over an element $K \in \mathrm{T}_{h}$ yield to

$$
\begin{gathered}
\int_{K} \boldsymbol{\sigma} \cdot \bar{\tau} \mathrm{d} x-\int_{K} u \operatorname{div} \bar{\tau} \mathrm{d} x+\int_{\partial K} u \mathbf{n}_{K} \cdot \bar{\tau} \mathrm{d} s=0 \\
-\int_{K} \boldsymbol{\sigma} \cdot \nabla \bar{v} \mathrm{~d} x-k^{2} \int_{K} u \bar{v} \mathrm{~d} x+\int_{\partial K} \boldsymbol{\sigma} \cdot \mathbf{n}_{K} \bar{v} \mathrm{~d} s=\int_{K} f \bar{v} \mathrm{~d} x,
\end{gathered}
$$

where $\mathbf{n}_{K}$ denotes the unit outward normal vector to $\partial K$ and the overbar denotes complex conjugation. The mixed discontinuous Galerkin spaces $V_{h}$ and $\mathbf{Q}_{h}$ are defined as

$$
\begin{aligned}
& V_{h}=\left\{v \in L^{2}(\Omega) ;\left.v\right|_{K} \in P_{r}(K), \forall K \in \mathrm{T}_{h}\right\}, \\
& \mathbf{Q}_{h}=\left\{\boldsymbol{\tau} \in\left(L^{2}(\Omega)\right)^{d} ;\left.\boldsymbol{\tau}\right|_{K} \in\left(P_{l}(K)\right)^{d}, \forall K \in \mathrm{T}_{h}\right\},
\end{aligned}
$$

where $P_{r}(K)(r \geq 1)$ stands for the set of all polynomials of degree less than or equal to $r$ on $K$. Based on the weak formulations (7) and (8), the solution $\left(u_{h}, \sigma_{h}\right)$ of the mixed discontinuous Galerkin method can be defined by

$$
\begin{gathered}
\int_{K} \boldsymbol{\sigma}_{h} \cdot \bar{\tau}_{h} \mathrm{~d} x-\int_{K} u_{h} \operatorname{div} \bar{\tau}_{h} \mathrm{~d} x+\int_{\partial K} \widehat{u}_{K} \mathbf{n}_{K} \cdot \bar{\tau}_{h} \mathrm{~d} s=0 \\
-\int_{K} \boldsymbol{\sigma}_{h} \cdot \nabla \bar{v}_{h} \mathrm{~d} x-k^{2} \int_{K} u_{h} \bar{v}_{h} \mathrm{~d} x+\int_{\partial K} \widehat{\boldsymbol{\sigma}}_{K} \cdot \mathbf{n}_{K} \bar{v}_{h} \mathrm{~d} s=\int_{K} f \bar{v}_{h} \mathrm{~d} x,
\end{gathered}
$$

for any $\left(v_{h}, \tau_{h}\right) \in V_{h} \times \mathbf{Q}_{h}$, where $\widehat{u}_{K}$ and $\widehat{\sigma}_{K}$ are the numerical fluxes on the boundary $\partial K$. The numerical fluxes have to be suitably defined in order to ensure the stability and accuracy of the mixed discontinuous Galerkin method. Summing the above equations over all elements, $K \in \mathrm{T}_{h}$, and using the following integration by parts identity,

$$
\sum_{K \in \mathrm{T}_{h}} \int_{\partial K} v \bar{\tau} \cdot \mathbf{n d} s=\sum_{e \in \mathscr{E}_{h}} \int_{e}[v]\{\bar{\tau}\} \mathrm{d} s+\sum_{e \in \mathscr{C}_{h}^{I}} \int_{e}\{v\}[\bar{\tau}] \mathrm{d} s,
$$

we get

$$
\begin{gathered}
\left(\boldsymbol{\sigma}_{h}, \boldsymbol{\tau}_{h}\right)_{\Omega}-\left(u_{h}, \operatorname{div}_{h} \boldsymbol{\tau}_{h}\right)_{\Omega}+\sum_{e \in \mathscr{E}_{h}}\left\langle\left[\widehat{u}_{K}\right],\left\{\tau_{h}\right\}\right\rangle_{e} \\
+\sum_{e \in \mathscr{E}_{h}^{I}}\left\langle\left\{\widehat{u}_{K}\right\},\left[\boldsymbol{\tau}_{h}\right]\right\rangle_{e}=0,
\end{gathered}
$$

$$
\begin{aligned}
& \left(\operatorname{div}_{h} \boldsymbol{\sigma}_{h}, v_{h}\right)_{\Omega}-k^{2}\left(u_{h}, v_{h}\right)_{\Omega}+\sum_{e \in \mathscr{E}_{h}^{I}}\left\langle\left[\widehat{\sigma}_{K}-\boldsymbol{\sigma}_{h}\right]\left\{v_{h}\right\}\right\rangle_{e} \\
& +\sum_{e \in \mathscr{E}_{h}}\left\langle\left\{\widehat{\sigma}_{K}-\boldsymbol{\sigma}_{h}\right\},\left[v_{h}\right]\right\rangle_{e}=\left(f, v_{h}\right)_{\Omega} .
\end{aligned}
$$

Here, for any piecewise smooth scalar function $v$ and vector-valued function $\tau$, let $\left.\operatorname{div}_{h} \tau\right|_{K}=\left.\operatorname{div} \tau\right|_{K}$ on any element $K \in \mathrm{T}_{h}$. In this paper, we choose the numerical fluxes in (12) and (13) as follows: 


$$
\begin{aligned}
& \widehat{\sigma}_{h}=\left\{\boldsymbol{\sigma}_{h}\right\}, \widehat{u}_{h}=\left\{u_{h}\right\}+i \eta_{e} h_{e}^{-1}\left[\boldsymbol{\sigma}_{h}\right], \quad \text { on } e \in \mathscr{E}_{h}^{I}, \\
& \widehat{\sigma}_{h}=\boldsymbol{\sigma}_{h}, \widehat{u}_{h}=\frac{i}{k} \boldsymbol{\sigma}_{h} \cdot \mathbf{n}-\frac{i}{k} g, \quad \text { on } e \in \mathscr{E}_{h}^{B},
\end{aligned}
$$

where $\eta_{e}$ is positive constant. Substituting the numerical fluxes (14) and (15) into (12) and (13), we get the mixed discontinuous Galerkin formulation of (1) and (2) as follows: finding $\left(u_{h}, \sigma_{h}\right) \in V_{h} \times \mathbf{Q}_{h}$ such that

$$
\begin{aligned}
& \left(\boldsymbol{\sigma}_{h}, \boldsymbol{\tau}_{h}\right)_{\Omega}-\left(u_{h}, \operatorname{div}_{h} \boldsymbol{\tau}_{h}\right)_{\Omega}+\sum_{e \in \mathscr{E} I}\left\langle\left\{u_{h}\right\},\left[\boldsymbol{\tau}_{h}\right]\right\rangle_{e} \\
& \quad+\sum_{e \in \mathscr{E} I} i \eta_{e} h_{e}^{-1}\left\langle\left[\boldsymbol{\sigma}_{h}\right],\left[\boldsymbol{\tau}_{h}\right]\right\rangle_{e} \\
& \quad+\frac{i}{k}\left\langle\boldsymbol{\sigma}_{h} \cdot \mathbf{n}, \boldsymbol{\tau}_{h} \cdot \mathbf{n}\right\rangle_{\Gamma}=\frac{i}{k}\left\langle g, \boldsymbol{\tau}_{h} \cdot \mathbf{n}\right\rangle_{\Gamma} \\
& \left(\operatorname{div}_{h} \boldsymbol{\sigma}_{h}, v_{h}\right)_{\Omega}-k^{2}\left(u_{h}, v_{h}\right)_{\Omega}-\sum_{e \in \mathscr{E} I}\left\langle\left[\boldsymbol{\sigma}_{h}\right],\left\{v_{h}\right\}\right\rangle_{e}=\left(f, v_{h}\right)_{\Omega},
\end{aligned}
$$

for any $\left(v_{h}, \tau_{h}\right) \in V_{h} \times \mathbf{Q}_{h}$.

\section{Numerical Experiments}

In this section, we present some numerical examples of the mixed discontinuous Galerkin method. In the following experiments, we set penalty parameter $\eta_{e}=10$.

3.1. Example 1: Helmholtz Equation in Convex Domain. We first consider a Helmholtz problem defined on the domain $\Omega=[-0.5,0.5] \times[-0.5,0.5]$, and the exact solution is given by

$$
\begin{aligned}
& u=\frac{\cos (k r)}{k}-\frac{\cos k+i \sin k}{k\left(J_{0}(k)+i J_{1}(k)\right)} J_{0}(k r), \\
& f=\frac{\sin (k r)}{r},
\end{aligned}
$$

where $r=\sqrt{x^{2}+y^{2}}$ and $J_{n}(z)$ are the Bessel functions of the first kind and order $n$. The function $g$ is chosen by the exact solution. We test uniformly triangular meshes as shown in Figure 1(a). The numerical errors and convergence rate for $k=5$ and $k=10$ with the discontinuous Galerkin pair $\mathbf{P}_{p+1}^{-1}-P_{p}^{-1}(p=0,1)$ are given in Tables $1-4$. We can observe that the convergence rate is optimal for the flux variable in both $H$ (div)-like norm and $L^{2}$ norm and the scattered field in $L^{2}$ norm.

\subsection{Example 2: Helmholtz Equation in Nonconvex Domain.} In this numerical test, we solve the Helmholtz problem defined on a nonconvex domain as shown in Figure 1(b). We take $f=0$ and choose the exact solution as follows:

$$
u=J_{\xi}\left(k \sqrt{(x+1)^{2}+y^{2}}\right) \cos \left(\xi \arctan \left(\frac{y}{x+1}\right)\right) .
$$

The boundary condition $g$ is given by the exact solution $u$ for $\xi=1, \xi=3 / 2$, and $\xi=2 / 3$. We can check that $u$ is smooth for $\xi \in \mathbb{N}$, while its derivative has a singularity at $(-1,0)$ for $\xi \notin \mathbb{N}$. For $\xi=1,3 / 2$, and $2 / 3$ and wave number $k=4$, Figures 2 and 3 show the errors of the mixed discontinuous Galerkin method with the finite element pair $\mathbf{P}_{1}^{-1}-P_{0}^{-1}$ and $\mathbf{P}_{2}^{-1}-P_{1}^{-1}$, respectively. It shows that convergent rates are optimal in three errors for cases $\xi=1$. We lose the optimal convergent in the flux variable $\left\|\sigma-\sigma_{h}\right\|_{0, \Omega}$, while the scalar variable $\left\|u-u_{h}\right\|_{0, \Omega}$ and the flux variable $\left\|\operatorname{div}\left(\sigma-\sigma_{h}\right)\right\|_{0, \Omega}$ still converge at the optimal order, for the case $\xi=2 / 3$ and $\xi=3 / 2$.

\subsection{Example 3: Helmholtz Equation with Large Wave Number.} This test is devoted to studying the numerical test for the same setting as example 1 with a large wave number. We solve the Helmholtz problem with different mesh sizes for four wave numbers $k=5,10,50$, and 100. The errors in $\left\|u-u_{h}\right\|_{0, \Omega},\left\|\sigma-\sigma_{h}\right\|_{0, \Omega}$, and $\left\|\operatorname{div}\left(\sigma-\sigma_{h}\right)\right\|_{0, \Omega}$ are shown in Figure 4 with $p=0$ and Figure 5 with $p=1$. It indicates that the mixed discontinuous Galerkin method is convergent for the cases $k=5$ and $k=10$, and the errors begin to reduce as $h$ becomes to be quite small for large wave numbers $k=50$ and 100. The surface plots of the mixed discontinuous Galerkin solution and the exact solution are shown in Figure 6. It shows that the mixed discontinuous Galerkin solution has the correct shape.

3.4. Example 4: Helmholtz Equation with Perturbation. We consider the Helmholtz equation with perturbation [14] in the following form:

$$
\begin{aligned}
-\Delta u-k^{2}(1+q(x)) u & =f, \quad \text { in } \Omega \in \mathbf{R}^{2}, \\
\frac{\partial u}{\partial n_{\Omega}}+i k u & =g, \quad \text { on } \Gamma=\partial \Omega,
\end{aligned}
$$

where $f=k^{2} q(x) u_{i}(x), g=-\left(\partial u_{i} / \partial n_{\Omega}\right)-\mathrm{i} k u_{i}, \quad u_{i}(x)=$ $\exp (i k x)$ is the incident field and the perturbation function $q(x)$ has compact support in the domain $\Omega$. Note that the solution is propagating for $q(x)>-1$, while it is evanescent for $q(x)<-1$. The numerical fluxes for the Helmholtz equation with perturbation are the same as (14) and (15). In this test, let $\Omega=[-1.5,1.5] \times[-1.5,1.5]$. Due to the area of compact support of perturbation $q(x)$ which is little relative to the entire area $\Omega$, with the aim of better observing the experimental phenomena of the scattered field, we limit the interval to $[-1,1] \times[-1,1]$ as shown in Figures 7 and 8 .

(i) Take the perturbation function $q(\mathbf{x})=1.5 \mathrm{exp}$ $\left(-160|\mathbf{x}|^{2}\right)$ with wave number $k=40$. The graphics of the real part of the scattered field and perturbation function is shown in Figure 7. We can find that the wave propagates through the origin of the positive perturbation function.

(ii) In Figure 8, we present the geometry of the real part of the scattered field and perturbation function with the perturbation function $q(\mathbf{x})=-1.5 \exp \left(-160|\mathbf{x}|^{2}\right)$ and wave number $k=40$. The results show that due 




(a)

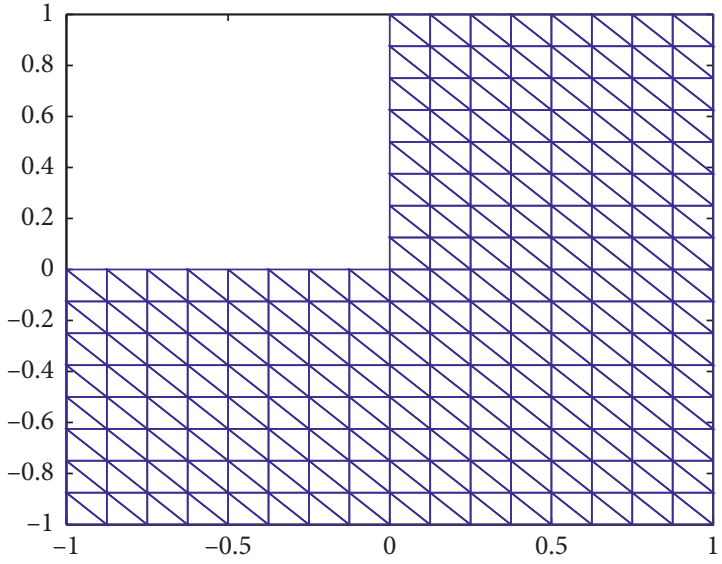

(b)

Figure 1: The meshes for example 3.1 (a) and example 3.2 (b).

TABLe 1: Numerical errors and convergence rate with $k=5$ for the $\mathbf{P}_{1}^{-1}-P_{0}^{-1}$ element.

\begin{tabular}{lccccc}
\hline$h$ & $\left\|u-u_{h}\right\|_{0}$ & Rate & $\left\|\sigma-\sigma_{h}\right\|_{0}$ & Rate & $\left\|\operatorname{div}\left(\sigma-\sigma_{h}\right)\right\|_{0}$ \\
\hline $1 / 2$ & $1.0505 e-01$ & - & $4.0575 e-01$ & - & $2.6311 e+00$ \\
$1 / 4$ & $5.2449 e-02$ & 1.0020 & $1.3732 e-01$ & 1.5631 & $1.3206 e+00$ \\
$1 / 8$ & $2.7299 e-02$ & 0.9421 & $3.8612 e-02$ & 1.8304 & $6.8713 e-01$ \\
$1 / 16$ & $1.3851 e-02$ & 0.9788 & $9.9766 e-03$ & 1.9524 & $3.4859 e-01$ \\
$1 / 32$ & $6.9536 e-03$ & 0.9942 & $2.5156 e-03$ & 1.9877 & $1.7499 e-01$ \\
$1 / 64$ & $3.4804 e-03$ & 0.9985 & $6.3026 e-04$ & 1.9969 & $8.7586 e-02$ \\
\hline
\end{tabular}

TABLE 2: Numerical errors and convergence rates with $k=10$ for the $\mathbf{P}_{1}^{-1}-P_{0}^{-1}$ element.

\begin{tabular}{lccccc}
\hline$h$ & $\left\|u-u_{h}\right\|_{0}$ & Rate & $\left\|\sigma-\sigma_{h}\right\|_{0}$ & Rate & $\left\|\operatorname{div}\left(\sigma-\sigma_{h}\right)\right\|_{0}$ \\
\hline $1 / 2$ & $5.4580 e-02$ & - & $9.9906 e-01$ & - & $8.9505 e+00$ \\
$1 / 4$ & $2.1681 e-02$ & 1.3319 & $5.0097 e-01$ & 0.9958 & $3.2653 e+00$ \\
$1 / 8$ & $1.8045 e-02$ & 0.2648 & $2.1682 e-01$ & 1.2082 & $1.5504 e+00$ \\
$1 / 16$ & $1.2249 e-02$ & 0.5590 & $6.3883 e-02$ & 1.7630 & $1.1230 e+00$ \\
$1 / 32$ & $6.6122 e-03$ & 0.8894 & $1.6672 e-02$ & 1.9381 & $6.1967 e-01$ \\
$1 / 64$ & $3.3701 e-03$ & 0.9723 & $4.2134 e-03$ & 1.9843 & $3.1747 e-01$ \\
\hline
\end{tabular}

TABLe 3: Numerical errors and convergence rates with $k=5$ for the $\mathbf{P}_{1}^{-1}-P_{0}^{-1}$ element.

\begin{tabular}{lccccc}
\hline$h$ & $\left\|u-u_{h}\right\|_{0}$ & Rate & $\left\|\sigma-\sigma_{h}\right\|_{0}$ & Rate & $\left\|\operatorname{div}\left(\sigma-\sigma_{h}\right)\right\|_{0}$ \\
\hline $1 / 2$ & $2.1579 e-02$ & - & $4.3274 e-02$ & - & $5.7183 e-01$ \\
$1 / 4$ & $6.6959 e-03$ & 1.6883 & $7.0819 e-03$ & 2.6113 & $1.8744 e-01$ \\
$1 / 8$ & $1.7359 e-03$ & 1.9476 & $8.8260 e-04$ & 3.0043 & $4.8484 e-02$ \\
$1 / 16$ & $4.3740 e-04$ & 1.9886 & $1.1042 e-04$ & 2.9987 & $1.2215 e-02$ \\
$1 / 32$ & $1.0956 e-04$ & 1.9973 & $1.3799 e-05$ & 3.0003 & $3.0595 e-03$ \\
$1 / 64$ & $2.7402 e-05$ & 1.9993 & $1.5553 e-06$ & 3.1493 & $7.6522 e-04$ \\
\hline
\end{tabular}

TABLE 4: Numerical errors and convergence rates with $k=10$ for the $\mathbf{P}_{1}^{-1}-P_{0}^{-1}$ element.

\begin{tabular}{lccccc}
\hline$h$ & $\left\|u-u_{h}\right\|_{0}$ & Rate & $\left\|\sigma-\sigma_{h}\right\|_{0}$ & Rate & $\left\|\operatorname{div}\left(\sigma-\sigma_{h}\right)\right\|_{0}$ \\
\hline $1 / 2$ & $4.3837 e-02$ & - & $5.3549 e-01$ & - & $4.7389 e+00$ \\
$1 / 4$ & $1.4489 e-02$ & 1.5972 & $7.2364 e-02$ & 2.8875 & $1.2945 e+00$ \\
$1 / 8$ & $4.1745 e-03$ & 1.7953 & $6.4364 e-03$ & 3.4909 & $3.9645 e-01$ \\
$1 / 16$ & $1.0708 e-03$ & 1.9629 & $8.4948 e-04$ & 2.9216 & $1.0206 e-01$ \\
$1 / 32$ & $2.6887 e-04$ & 1.9937 & $1.1549 e-04$ & 2.8788 & $2.5641 e-02$ \\
$1 / 64$ & $6.7280 e-05$ & 1.9986 & $1.3821 e-05$ & 3.0629 & $6.4172 e-03$ \\
\hline
\end{tabular}






(a)



$$
\begin{aligned}
& \multimap \quad \xi=1 \\
& \rightarrow-\xi=3 / 2
\end{aligned}
$$$$
\text { - }+-\xi=2 / 3
$$

(b)



(c)

Figure 2: Convergence rates with $k=4$ and $\xi=1,2 / 3$, and $3 / 2$ for the $\mathbf{P}_{1}^{-1}-P_{0}^{-1}$ element. (a) $\left\|u-u_{h}\right\|_{0}$. (b) $\left\|\sigma-\sigma_{h}\right\|_{0}$. (c) $\left\|\operatorname{div}\left(\sigma-\sigma_{h}\right)\right\|_{0}$.

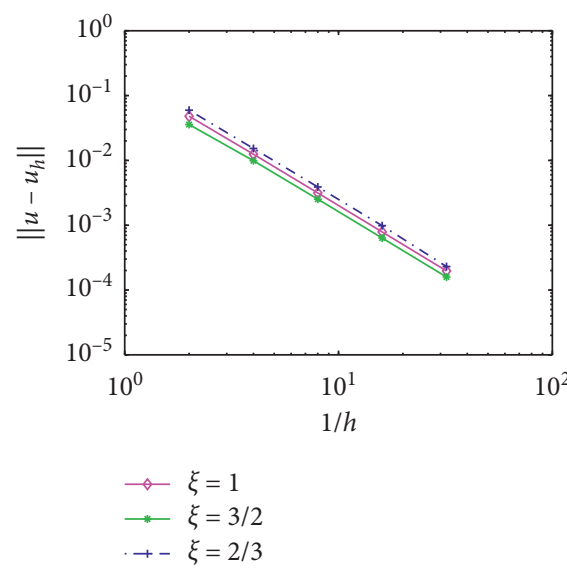

(a)

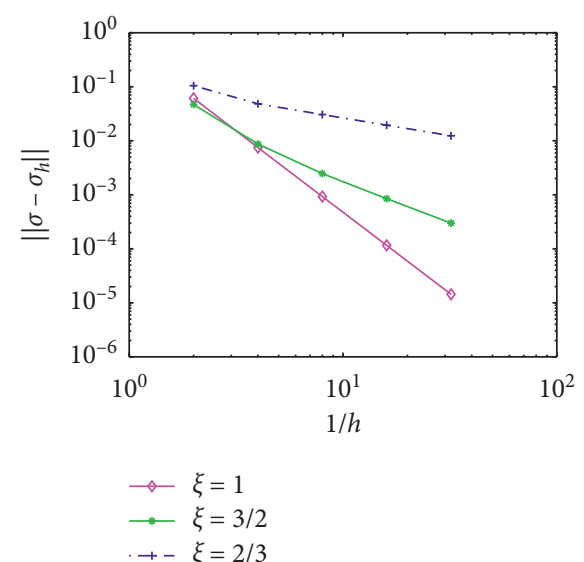

(b)



(c)

Figure 3: Convergence rates with $k=4$ and $\xi=1,2 / 3$, and $3 / 2$ for the $\mathbf{P}_{2}^{-1}-P_{1}^{-1}$ element. (a) $\left\|u-u_{h}\right\|_{0}$. (b) $\left\|\sigma-\sigma_{h}\right\|_{0}$. (c) $\left\|\operatorname{div}\left(\sigma-\sigma_{h}\right)\right\|_{0}$. 


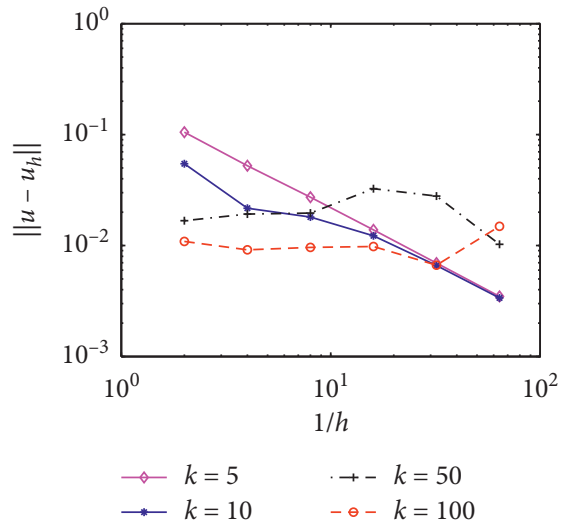

(a)

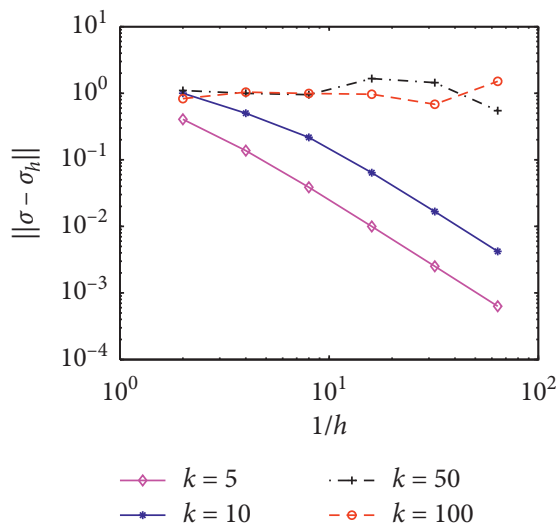

(b)



(c)

Figure 4: The errors for four wave numbers $k=5,10,50$, and 100 and $\mathbf{P}_{1}^{-1}-P_{0}^{-1}$. (a) $\left\|u-u_{h}\right\|_{0}$. (b) $\left\|\sigma-\sigma_{h}\right\|_{0}$. (c) $\left\|\operatorname{div}\left(\sigma-\sigma_{h}\right)\right\|_{0}$.

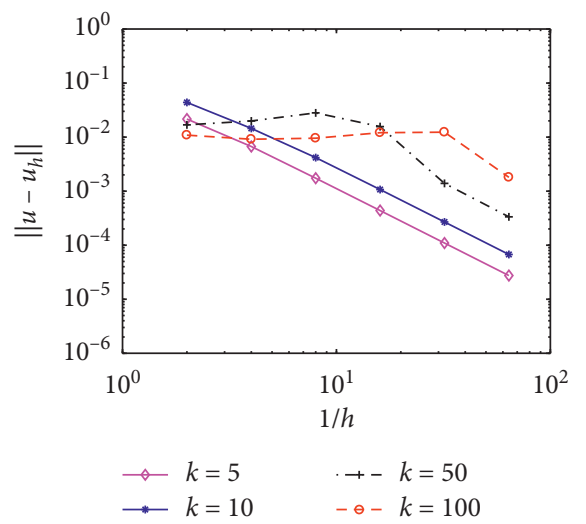

(a)



(b)

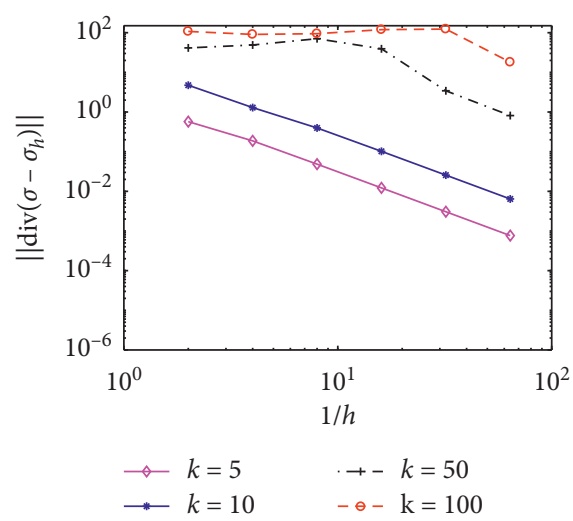

(c)

Figure 5: The errors for four wave numbers $k=5,10,50$, and 100 and $\mathbf{P}_{2}^{-1}-P_{1}^{-1}$. (a) $\left\|u-u_{h}\right\|_{0}$. (b) $\left\|\sigma-\sigma_{h}\right\|_{0}$. (c) $\left\|\operatorname{div}\left(\sigma-\sigma_{h}\right)\right\|_{0}$. 


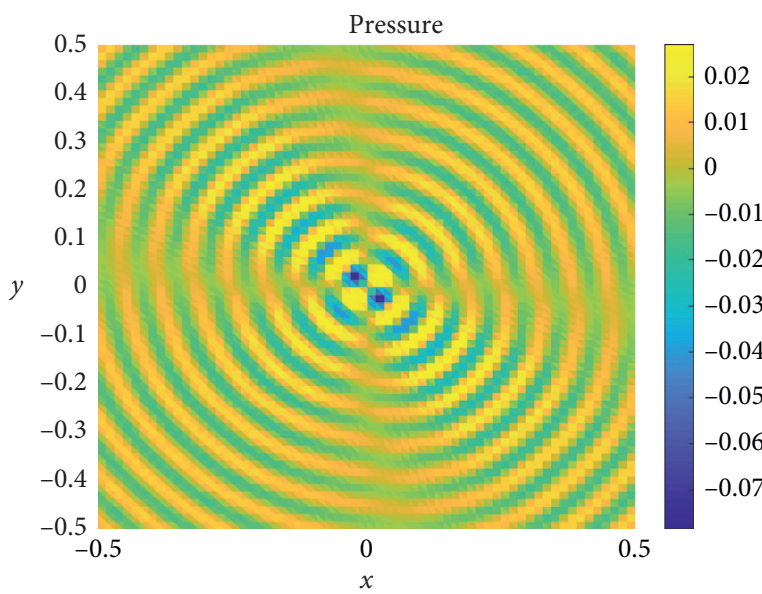

(a)

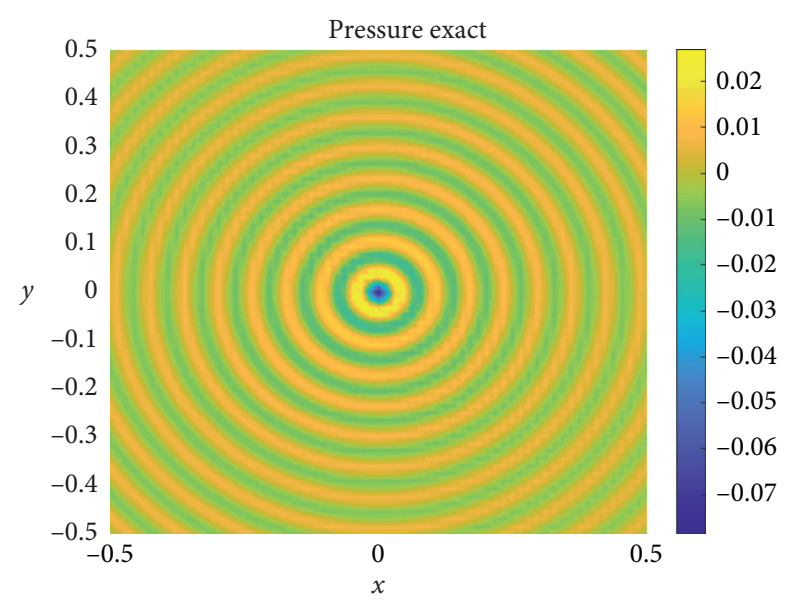

(b)

Figure 6: The surface plot for $k=100, h=1 / 64$, and $\mathbf{P}_{1}^{-1}-P_{0}^{-1}$. (a) Numerical solution. (b) Exact solution.



(a)

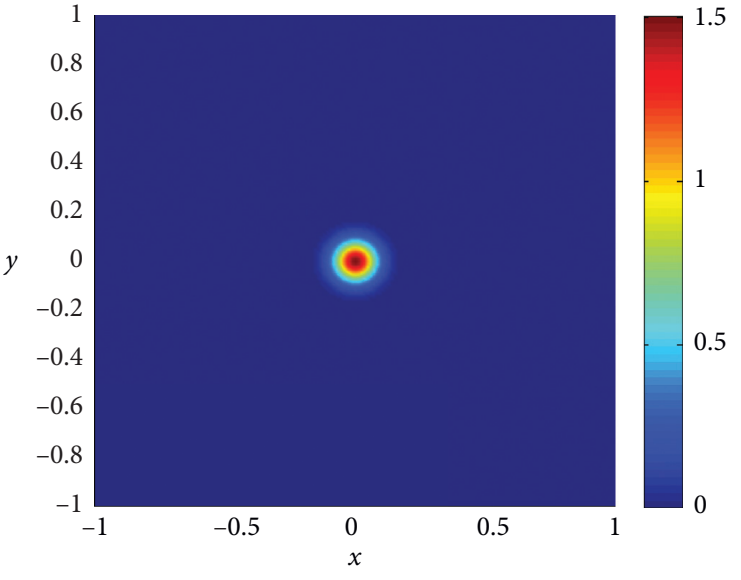

(b)

Figure 7: Scattered field and perturbation function with $k=40, h=1 / 256$, and $\mathbf{P}_{1}^{-1}-P_{0}^{-1}$. (a) Real part of the scattered field. (b) Perturbation function.

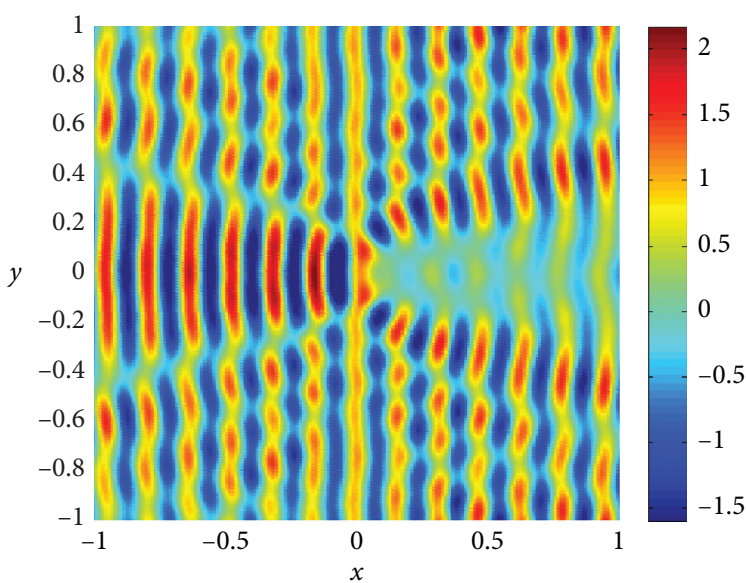

(a)

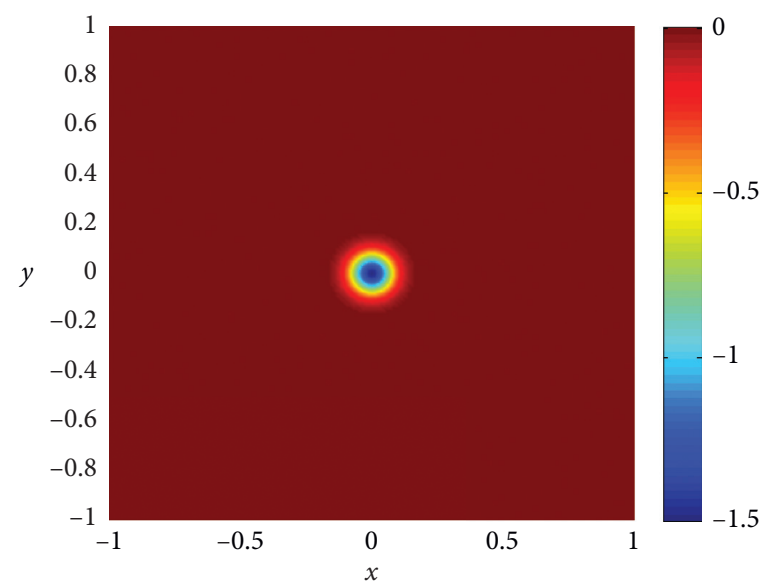

(b)

Figure 8: Scattered field and perturbation function with $k=40, h=1 / 256$, and $\mathbf{P}_{1}^{-1}-P_{0}^{-1}$. (a) Real part of he scattered field. (b) Perturbation function. 


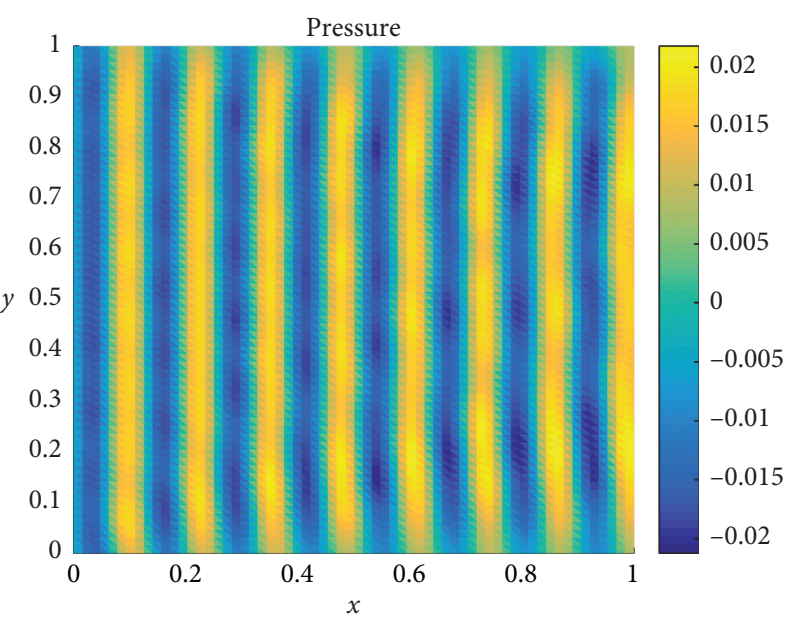

(a)



(b)

Figure 9: Real part of the waveguide solution with $\mathbf{P}_{1}^{-1}-P_{0}^{-1}$. (a) $k=16 \pi, h=1 / 64$. (b) $k=64 \pi, h=1 / 128$.

to the scattering of the plane wave impinged on the negative perturbation, the wave gradually disappears near the origin.

3.5. Example 5: Rectangular Waveguide. In this example, we consider the domain $\Omega$ is $\Omega=[0,1] \times[0,1]$, and the $2 \mathrm{D}$ rectangular waveguide model problem satisfies the Helmholtz equation (1) with the following boundary conditions:

$$
\begin{aligned}
& \frac{\partial u}{\partial \mathbf{n}}=0, \quad \text { on } \Gamma_{1}=(0,1) \times\{0\} \text { and } \Gamma_{3}=(0,1) \times\{1\} \\
& \frac{\partial u}{\partial \mathbf{n}}=g_{2}, \quad \text { on } \Gamma_{4}=\{0\} \times(0,1), \\
& \frac{\partial u}{\partial \mathbf{n}}=-i k u, \quad \text { on } \Gamma_{4}=\{1\} \times(0,1) .
\end{aligned}
$$

From $[3,22]$, we set in (1) and $g_{2}=e^{-(1 / 2)(y-1 / 2)^{2}}$ in (21). Similarly, we define the numerical fluxes for the rectangular waveguide (1) and (20) and (22) as the following:

$$
\begin{aligned}
& \widehat{\sigma}_{h}=\left\{\sigma_{h}\right\}, \widehat{u}_{h}=\left\{u_{h}\right\}+i \eta_{e} h_{e}^{-1}\left[\sigma_{h}\right], \quad \text { on } e \in \varepsilon_{h}^{I}, \\
& \widehat{\sigma}_{h}=\sigma_{h}, \widehat{u}_{h}=-\frac{i}{k} \sigma_{h} \cdot \mathbf{n}, \quad \text { on } e \in \Gamma_{2}, \\
& \widehat{\sigma}_{h}=-g_{2} \mathbf{n}, \widehat{u}_{h}=u_{h}, \quad \text { on } e \in \Gamma_{4}, \\
& \widehat{\sigma}_{h}=0, \widehat{u}_{h}=u_{h}, \quad \text { on } e \in \Gamma_{1} \cup \Gamma_{3} .
\end{aligned}
$$

The real part of the waveguide solutions for $k=16 \pi, h=$ $1 / 64$ and $k=64 \pi, h=1 / 128$ is shown in Figure 9, which is consistent with the results in $[3,22]$.

\section{Conclusion}

The paper developed the mixed discontinuous Galerkin method for the Helmholtz equation. We define the numerical fluxes and introduce the mixed Galerkin method.
Compared with [18], we set penalty parameter $\beta=0$ in numerical fluxes and use the discontinuous $\mathbf{P}_{P+1}^{-1}-P_{p}^{-1}(p \geq 0)$ finite element pair to approximate the flux variable and the scattered field. We present several numerical experiments to validate the effectiveness of the mixed discontinuous Galerkin method. The optimal convergence for the scattered field and the flux variable in the $L^{2}$ norm and the flux variable in the $H$ (div)-like norm is obtained numerically. Furthermore, we test the Helmholtz equation with a large wave number and the Helmholtz equation with perturbation function and the rectangular waveguide, which indicate the well performance of the mixed discontinuous Galerkin method.

\section{Data Availability}

The data used to support the findings of this study are available from the corresponding author upon request.

\section{Conflicts of Interest}

The authors declare that they have no conflicts of interest.

\section{Acknowledgments}

This study was supported by the Major Research and Development Program of China (grant no. 2016YFB0200901) and the National Natural Science Foundation of China (grant nos. 11771348 and 11801143).

\section{References}

[1] B. Engquist and A. Majda, "Radiation boundary conditions for acoustic and elastic wave calculations," Communications on Pure and Applied Mathematics, vol. 32, no. 3, pp. 313-357, 1979.

[2] K. Wang and Y. S. Wong, "Pollution-free finite difference schemes for non-homogeneous Helmholtz equation," International Journal of Numerical Analysis and Modeling, vol. 11, pp. 787-815, 2014. 
[3] K. Wang and Y. S. Wong, "Is pollution effect of finite difference schemes avoidable for multi-dimensional Helmholtz equations with high wave numbers?" Communications in Computational Physics, vol. 21, no. 2, pp. 490-514, 2017.

[4] K. Wang, Y. S. Wong, and J. Deng, "Efficient and accurate numerical solutions for Helmholtz equation in polar and spherical coordinates," Communications in Computational Physics, vol. 17, no. 3, pp. 779-807, 2015.

[5] K. Wang, Y. S. Wong, and J. Huang, "Analysis of pollutionfree approaches for multi-dimensional Helmholtz equations," International Journal of Numerical Analysis and Modeling, vol. 16, pp. 412-435, 2019.

[6] F. Ihlenburg and I. Babuška, "Finite element solution of the Helmholtz equation with high wave number part I. The $h$-version of the FEM," Computers \& Mathematics with Applications, vol. 30, no. 9, pp. 9-37, 1995.

[7] F. Ihlenburg and I. Babuska, "Finite element solution of the Helmholtz equation with high wave number. II. The $h-p$ version of the FEM," SIAM Journal on Numerical Analysis, vol. 34, no. 1, pp. 315-358, 1997.

[8] P. J. Harris, "A boundary element method for the Helmholtz equation using finite part integration," Computer Methods in Applied Mechanics and Engineering, vol. 95, no. 3, pp. 331342, 1992.

[9] Y. Du and Z. Zhang, "A numerical analysis of the weak Galerkin method for the Helmholtz equation with high wave number," Communications in Computational Physics, vol. 22, no. 1, pp. 133-156, 2017.

[10] L. Mu, J. Wang, and X. Ye, "A new weak Galerkin finite element method for the Helmholtz equation," IMA Journal of Numerical Analysis, vol. 35, no. 3, pp. 1228-1255, 2015.

[11] L. Mu, J. Wang, X. Ye, and S. Zhao, "A numerical study on the weak Galerkin method for the Helmholtz equation," Communications in Computational Physics, vol. 15, no. 5, pp. 1461-1479, 2014.

[12] R. Wang, X. Wang, Q. Zhai, and K. Zhang, "A weak Galerkin mixed finite element method for the Helmholtz equation with large wave numbers," Numerical Methods for Partial Differential Equations, vol. 34, no. 3, pp. 1009-1032, 2018.

[13] O. Z. Mehdizadeh and M. Paraschivoiu, "Investigation of a two-dimensional spectral element method for Helmholtz's equation," Journal of Computational Physics, vol. 189, no. 1, pp. 111-129, 2003.

[14] H. Wang, W. Yang, and Y. Huang, "Adaptive finite element method for the sound wave problems in two kinds of media," Computers \& Mathematics with Applications, vol. 79, no. 3, pp. 789-801, 2020.

[15] X. Feng and H. Wu, "Discontinuous Galerkin methods for the Helmholtz equation with large wave number," SIAM Journal on Numerical Analysis, vol. 47, no. 4, pp. 2872-2896, 2009.

[16] H. Chen, P. Lu, and X. Xu, "A hybridizable discontinuous Galerkin method for the Helmholtz equation with high wave number," SIAM Journal on Numerical Analysis, vol. 51, no. 4, pp. 2166-2188, 2013.

[17] R. Griesmaier and P. Monk, "Error analysis for a hybridizable discontinuous Galerkin method for the Helmholtz equation," Journal of Scientific Computing, vol. 49, no. 3, pp. 291-310, 2011.

[18] X. Feng and Y. Xing, "Absolutely stable local discontinuous Galerkin methods for the Helmholtz equation with large wave number," Mathematics of Computation, vol. 82, no. 283, pp. 1269-1296, 2012.

[19] D. N. Arnold, F. Brezzi, B. Cockburn, and L. D. Marini, "Unified analysis of discontinuous Galerkin methods for elliptic problems," SIAM Journal on Numerical Analysis, vol. 39, no. 5, pp. 1749-1779, 2002.

[20] F. Brezzi, G. Manzini, D. Marini, P. Pietra, and A. Russo, "Discontinuous Galerkin approximations for elliptic problems," Numerical Methods for Partial Differential Equations, vol. 16, no. 4, pp. 365-378, 2000.

[21] Q. Hong, F. Wang, S. Wu, and J. Xu, "A unified study of continuous and discontinuous Galerkin methods," Science China Mathematics, vol. 62, no. 1, pp. 1-32, 2019.

[22] M. Bollhöfer, M. J. Grote, and O. Schenk, "Algebraic multilevel preconditioner for the Helmholtz equation in heterogeneous media," SIAM Journal on Scientific Computing, vol. 31, no. 5, pp. 3781-3805, 2009. 